\title{
ON A THIN SET OF INTEGERS INVOLVING THE LARGEST PRIME FACTOR FUNCTION
}

\section{JEAN-MARIE DE KONINCK and NICOLAS DOYON}

\author{
Received 22 April 2002
}

\begin{abstract}
For each integer $n \geq 2$, let $P(n)$ denote its largest prime factor. Let $S:=$ $\{n \geq 2: n$ does not divide $P(n) !\}$ and $S(x):=\#\{n \leq x: n \in S\}$. Erdős (1991) conjectured that $S$ is a set of zero density. This was proved by Kastanas (1994) who established that $S(x)=O(x / \log x)$. Recently, Akbik (1999) proved that $S(x)=$ $O(x \exp \{-(1 / 4) \sqrt{\log x}\})$. In this paper, we show that $S(x)=x \exp \{-(2+o(1)) \times$ $\sqrt{\log x \log \log x}$. We also investigate small and large gaps among the elements of $S$ and state some conjectures.
\end{abstract}

2000 Mathematics Subject Classification: 11B05, 11N25.

1. Introduction. For each integer $n \geq 2$, let $P(n)$ denote its largest prime factor and let

$$
S:=\{n \geq 2: n \text { does not divide } P(n) !\}, \quad S(x):=\#\{n \leq x: n \in S\} .
$$

Thus, the first 25 elements of $S$ are

$$
\begin{gathered}
4,8,9,12,16,18,24,25,27,32,36,45,48,49 \\
50,54,64,72,75,80,81,90,96,98,100
\end{gathered}
$$

while using a computer, we easily obtain that $S(10)=3, S(100)=25, S(1000)=$ $127, S\left(10^{4}\right)=593, S\left(10^{5}\right)=2806, S\left(10^{6}\right)=13567, S\left(10^{7}\right)=67252$, and $S\left(10^{8}\right)$ $=342022$.

In 1991, Erdős [2] challenged his readers to prove that $S$ is a set of zero density. In 1994, Kastanas [4] proved that result, while K. Ford (see [4]) observed that $S(x)=O(x / \log x)$. In 1999, Akbik [1] proved that $S(x)=O(x \exp \{-(1 / 4)$ $\times \sqrt{\log x}\}$.

Our main goal here is to prove that

$$
S(x)=x \exp \{-(2+o(1)) \sqrt{\log x \log \log x}\}
$$


In order to prove (1.3), we establish the following two bounds valid for each fixed $\delta>0$ :

$$
\begin{aligned}
& S(x) \gg x \exp \{-2(1+\delta) \sqrt{\log x \log \log x}\}, \\
& S(x) \ll x \exp \{-2(1-\delta) \sqrt{\log x \log \log x}\} .
\end{aligned}
$$

Finally, we investigate small and large gaps among the elements of $S$ and state some conjectures.

2. The lower bound for $S(x)$. Let $\delta>0$ be small and fixed. Since every integer $n \geq 2$ divisible by the square of its largest prime factor must belong to $S$, we have that

$$
S(x) \geq \sum_{p \leq \sqrt{x}} \sum_{\substack{m p^{2} \leq x \\ P(m) \leq p}} 1=\sum_{p \leq \sqrt{x}} \sum_{\substack{m \leq x / p^{2} \\ P(m) \leq p}} 1=\sum_{p \leq \sqrt{x}} \Psi\left(\frac{x}{p^{2}}, p\right),
$$

where $\Psi(x, y):=\#\{n \leq x: P(n) \leq y\}$.

Setting $u=\log x / \log y$, we recall Hildebrand's estimate [3]

$$
\Psi(x, y)=x \rho(u)\left\{1+O\left(\frac{\log (u+1)}{\log y}\right)\right\}
$$

which holds for

$$
\exp \left\{(\log \log x)^{5 / 3+\varepsilon}\right\} \leq y \leq x,
$$

where $\varepsilon>0$ is any fixed real number, and where $\rho$ stands for Dickman's function whose asymptotic behaviour is given by

$$
\rho(u)=\exp \left\{-u\left(\log u+\log \log u-1+O\left(\frac{\log \log u}{\log u}\right)\right)\right\} \quad(u \rightarrow \infty)
$$

It follows from this last estimate that if $u$ is sufficiently large, then

$$
\log \rho(u) \geq-(1+\delta) u \log u
$$

Hence, if we choose $r$ sufficiently large, say $r \geq r_{0} \geq 2$, then for each $y \leq x^{1 / r}$, we have $u=\log x / \log y \geq r$, thereby guaranteeing the validity of (2.5). 
Therefore, it follows from (2.4) and (2.5) that, with $u=\log \left(x / p^{2}\right) / \log p=$ $\log x / \log p-2$,

$$
\log \rho(u) \geq-(1+\delta) \frac{\log x}{\log p} \log \log x \quad\left(u \geq r_{0}\right)
$$

and hence (2.1) and (2.2) yield

$$
\begin{aligned}
S(x) & \gg x \sum_{e^{(\log \log x)^{5 / 3+\varepsilon}} \leq p \leq x^{1 / r}} \frac{1}{p^{2} e^{(1+\delta)(\log x / \log p) \log \log x}} \\
& =x \int_{e^{(\log \log x)^{5 / 3+\varepsilon}}}^{x^{1 / r}} \frac{d \pi(t)}{t^{2} \cdot e^{(1+\delta)(\log x / \log t) \log \log x}},
\end{aligned}
$$

where $\pi(t)$ stands for the number of primes not exceeding $t$. Now, set

$$
L_{\delta}(x):=\sqrt{(1+\delta) \log x \log \log x} \quad(x \geq 3)
$$

so that, for any $\delta_{1}>0$, we have, for $x$ sufficiently large,

$$
\left[L_{\delta}(x),\left(1+\delta_{1}\right) L_{\delta}(x)\right] \subset\left[(\log \log x)^{5 / 3+\varepsilon}, \frac{1}{r} \log x\right]
$$

Using this, it follows from (2.7) that setting $J(x):=\left[e^{L_{\delta}(x)}, e^{\left(1+\delta_{1}\right) L_{\delta}(x)}\right]$,

$$
\begin{aligned}
S(x) & \gg x \int_{t \in J(x)} \frac{d \pi(t)}{t^{2} \cdot e^{(1+\delta)(\log x / \log t) \log \log x}} \\
& >x \min _{t \in J(x)}\left(\frac{1}{t^{2} \cdot e^{(1+\delta)(\log x / \log t) \log \log x}}\right) \int_{t \in J(x)} d \pi(t) .
\end{aligned}
$$

Now, observe that since $t / \log t<\pi(t)<2(t / \log t)$ for $t \geq 11$, we have that

$$
\begin{aligned}
\int_{t \in J(x)} d \pi(t) & =\pi\left(e^{\left(1+\delta_{1}\right) L_{\delta}(x)}\right)-\pi\left(e^{L_{\delta}(x)}\right) \\
& >\frac{e^{\left(1+\delta_{1}\right) L_{\delta}(x)}}{\left(1+\delta_{1}\right) L_{\delta}(x)}-\frac{e^{L_{\delta}(x)}}{L_{\delta}(x)} \\
& \gg \frac{e^{\left(1+\delta_{1}\right) L_{\delta}(x)}}{\left(1+\delta_{1}\right) L_{\delta}(x)} .
\end{aligned}
$$


On the other hand, setting $v=\log t$ and afterwards $w=v / L_{\delta}(x)$, we have

$$
\begin{aligned}
\min _{t \in J(x)} & \left(\frac{1}{t^{2} \cdot e^{(1+\delta)(\log x / \log t) \log \log x}}\right) \\
& =\min _{L_{\delta}(x) \leq v \leq\left(1+\delta_{1}\right) L_{\delta}(x)}\left(\frac{1}{e^{2 v+(1+\delta)(\log x / v) \log \log x}}\right) \\
& =\min _{1 \leq w \leq 1+\delta_{1}}\left(\frac{1}{e^{2 w L_{\delta}(x)+(1+\delta)\left(\log x / w L_{\delta}(x)\right) \log \log x}}\right) \\
& =\min _{1 \leq w \leq 1+\delta_{1}}\left(\frac{1}{e^{(2 w+1 / w) L_{\delta}(x)}}\right) \\
& \gg \frac{1}{e^{\left(3+2 \delta_{1}\right) L_{\delta}(x)}}
\end{aligned}
$$

since $2 w+1 / w \leq 2+2 \delta_{1}+1=3+2 \delta_{1}$ for each $w \in\left[1,1+\delta_{1}\right]$.

Hence, using (2.11) and (2.12), it follows from (2.10) that

$$
\begin{aligned}
S(x) & \gg x \frac{e^{\left(1+\delta_{1}\right) L_{\delta}(x)}}{\left(1+\delta_{1}\right) L_{\delta}(x)} \cdot \frac{1}{e^{\left(3+2 \delta_{1}\right) L_{\delta}(x)}} \\
& =x \frac{e^{-\left(2+\delta_{1}\right) L_{\delta}(x)}}{\left(1+\delta_{1}\right) L_{\delta}(x)} \\
& \gg x e^{-2\left(1+\delta_{1}\right) L_{\delta}(x)},
\end{aligned}
$$

which establishes (1.4) by taking $\delta_{1}$ sufficiently small.

3. The upper bound for $S(x)$. First, we establish that

$$
S(x)<\sum_{2 \leq r<\log x / \log 2} \sum_{p<x^{1 / r}} \Psi\left(\frac{x}{p^{r}}, p r\right) .
$$

Actually, this inequality is based on a very simple observation; namely, the fact that if $n \in S$, then there exist a prime $p$ and an integer $r \geq 2$ such that $p^{r}$ divides $n$ but does not divide $P(n)$ !, in which case $P(n)<p r$. Hence, writing $n=p^{r} m$, we have that $P(m) \leq P(n)<p r$. These conditions imply that if $n \in S$ and $n \leq x$, then we have $r<\log x / \log 2, p<x^{1 / r}, m<x / p^{r}$, and $P(m)<p r$, thus proving (3.1).

We now move to find an upper bound for the inner sum on the right-hand side of (3.1); namely, $\sum_{p<x^{1 / r}} \Psi\left(x / p^{r}, p r\right)$, uniformly for all $r \geq 2$. For this purpose, we fix $r \geq 2$ and separate this sum on $p$ into three distinct sums as follows:

$$
\sum_{p<x^{1 / r}} \Psi\left(\frac{x}{p^{r}}, p r\right)=S_{1}(x)+S_{2}(x)+S_{3}(x),
$$


where the sums $S_{1}(x), S_{2}(x)$, and $S_{3}(x)$ run, respectively, in the following ranges:

$$
\begin{gathered}
p \leq \exp \left\{(\log \log x)^{2}\right\}, \\
\exp \left\{(\log \log x)^{2}\right\}<p \leq \exp \{2 \sqrt{\log x \log \log x}\}, \\
\exp \{2 \sqrt{\log x \log \log x}\}<p<x^{1 / r} .
\end{gathered}
$$

The first sum is negligible since it is clear that, using the well-known estimate,

$$
\Psi(X, Y) \ll X e^{-(1 / 2) \log X / \log Y} \quad(X \geq Y \geq 2)
$$

(see, e.g., Tenenbaum [5, Chapter III.5, Theorem 1]), we get that

$$
\begin{aligned}
S_{1}(x) & <\exp \left\{(\log \log x)^{2}\right\} \Psi\left(x, \frac{\log x}{\log 2} \exp \left\{(\log \log x)^{2}\right\}\right) \\
& \ll x e^{(-1 / 2+o(1))\left(\log x /(\log \log x)^{2}\right)} .
\end{aligned}
$$

The third one is also easily bounded since

$$
\begin{aligned}
S_{3}(x) & <\sum_{\exp \{2 \sqrt{\log x \log \log x}\}<p<x} \frac{x}{p^{r}} \\
& \ll x \sum_{p>\exp \{2 \sqrt{\log x \log \log x}\}} \frac{1}{p^{2}} \\
& \ll x \exp \{-2 \sqrt{\log x \log \log x}\} .
\end{aligned}
$$

To estimate $S_{2}(x)$, we use essentially the same technique as in the proof of (1.4).

First, it follows from (2.4) that

$$
\log \rho(u) \leq-u \log (u)
$$

provided $u$ is sufficiently large. Then, with the same approach as in the proof of (1.4), we get that, for each fixed integer $r \geq 2$,

$$
S_{2}(x) \ll x \int_{1}^{2 \sqrt{\log x \log \log x}} \frac{d v}{v^{r-1} e^{v+\log x \log \log x / v}} .
$$


Now, set $f(v)=v+\log x \log \log x / v$. Since $f^{\prime}(v)=1-\log x \log \log x / v^{2}$ and $f^{\prime}(v)=0$ when $v=v_{0}=\sqrt{\log x \log \log x}$, it is easy to see that $v_{0}$ is indeed a minimum for $f$. From this, it follows that

$$
\begin{aligned}
v+ & \frac{\log x \log \log x}{v} \\
& \geq f\left(v_{0}\right)=2 \sqrt{\log x \log \log x} \text { for each } v \in[1,2 \sqrt{\log x \log \log x}] .
\end{aligned}
$$

Using this in (3.8), we conclude that

$$
\begin{aligned}
S_{2}(x) & \ll x \exp \{-2 \sqrt{\log x \log \log x}\} \int_{1}^{2 \sqrt{\log x \log \log x}} \frac{d v}{v^{r-1}} \\
& \ll x \log (2 \sqrt{\log x \log \log x}) \exp \{-2 \sqrt{\log x \log \log x}\} .
\end{aligned}
$$

Combining (3.1), (3.2), (3.5), (3.6), and (3.10), we get (1.5).

4. Small and large gaps among elements of $S$. We can easily show that there are infinitely many $n \in S$ such that $n+1 \in S$. This follows from the fact that the Pell equation

$$
x^{2}-2 y^{2}=1
$$

has infinitely many solutions. Indeed, if $(x, y)$ is a solution of $(4.1)$, then by setting $n=2 y^{2}$ and $n+1=x^{2}$, we have that $P(n)^{2} \mid n$ and $P(n+1)^{2} \mid(n+1)$, in which case $n$ does not divide $P(n)$ ! and $n+1$ does not divide $P(n+1)$ !, which guarantees that $n, n+1 \in S$. In fact, if $T_{2}$ stands for the set of those $n \in S$ such that $n+1 \in S$ and if $T_{2}(x)=\#\left\{n \leq x: n \in T_{2}\right\}$, then it follows easily from the above that $T_{2}(x) \gg \log x$. In fact, most certainly, the true order of $T_{2}(x)$ is much larger than $\log x$, but we could not prove it.

It seems strange that such twin elements of $S$, that is, pairs of numbers $n$ and $n+1$ both in $S$, are more difficult to count than pairs of numbers $n$ and $n+4$ both in $S$. Indeed, if $F_{4}$ stands for the set of those $n \in S$ such that $n+4 \in S$ and if $F_{4}(x)=\#\left\{n \leq x: n \in F_{4}\right\}$, then we can show that

$$
F_{4}(x) \gg \frac{x^{1 / 4}}{\log x}
$$

Indeed, observe that given any prime $p$, then both numbers $n=p^{4}-4 p^{2}=$ $p^{2}\left(p^{2}-4\right)=p^{2}(p-2)(p+2)$ and $n+4=p^{4}-4 p^{2}+4=\left(p^{2}-2\right)^{2}$ belong to $S$. Since there are at least $\pi\left(x^{1 / 4}\right)$ such pairs up to $x$, estimate (4.2) follows from 
Chebychev's inequality $\pi(y) \gg y / \log y$. Finally, note that $T_{2}\left(10^{8}\right)=1175$, while $F_{4}\left(10^{8}\right)=1261$.

More generally, we conjecture that given any positive $k \geq 3$, the set $T_{k}:=$ $\{n \in S: n+1, n+2, \ldots, n+k-1 \in S\}$ is also an infinite set. We could not prove this to be true, even in the case where $k=3$. Note that the only numbers less than $10^{8}$ belonging to $T_{3}$ are $48,118579,629693,1294298,9841094$, and 40692424.

As for large gaps among consecutive elements of $S$, it follows from the fact that $S$ is a set of zero density that given any positive integer $k$, there are infinitely many integers $n$ such that the intervals $[n, n+k]$ contain no element of $S$. Table 4.1 gives, for each positive integer $k$, the smallest integer $n=n(k) \in S$ such that both $n$ and $n+100 k$ belong to $S$, while the open interval $(n, n+100 k)$ contains no element of $S$.

TABLE 4.1

\begin{tabular}{lrrrr}
\hline $100 k$ & $n=n(k)$ & & $100 k$ & $n=n(k)$ \\
\cline { 5 - 5 } 100 & 21025 & & 600 & 738606 \\
200 & 78408 & & 700 & 946832 \\
300 & 369303 & & 800 & 8000325 \\
400 & 1250256 & & 900 & 5382888 \\
500 & 1639078 & & 1000 & 5775000 \\
\hline
\end{tabular}

It is quite easy to show that

$$
n(k) \geq 2500 k^{2}-100 k+1 \text {. }
$$

Indeed, since all perfect squares belong to $S$ and since $(m+1)^{2}-m^{2}=2 m+$ 1 , it follows that the interval $(n, n+2 m+1)$ contains no element of $S$ and, therefore, that $n \geq m^{2}$. Hence, given a positive integer $k$, choose $m$ so that $100 k=2 m+2$, that is, $m=50 k-1$. Then, clearly, we have that $n(k) \geq m^{2}=$ $(50 k-1)^{2}$, which proves $(4.3)$.

It would also be interesting to obtain a decent upper bound for $n(k)$.

ACKNOWLEDGMENT. This research was supported in part by a grant from the Natural Sciences and Engineering Research Council (NSERC).

\section{REFERENCES}

[1] S. Akbik, On a density problem of Erdös, Int. J. Math. Math. Sci. 22 (1999), no. 3, 655-658.

[2] P. Erdős, Problem 6674, Amer. Math. Monthly 98 (1991), no. 10, 965.

[3] A. Hildebrand, On the number of positive integers $\leq x$ and free of prime factors $>y$, J. Number Theory 22 (1986), no. 3, 289-307.

[4] I. Kastanas, Solution to Problem 6674, Amer. Math. Monthly 101 (1994), no. 2, 179. 
[5] G. Tenenbaum, Introduction to Analytic and Probabilistic Number Theory, Cambridge Studies in Advanced Mathematics, vol. 46, Cambridge University Press, Cambridge, 1995.

Jean-Marie De Koninck: Département de Mathématiques et de Statistique, Université Laval, Québec, Québec, Canada G1K 7P4

E-mail address: jmdk@mat.u1ava1.ca

Nicolas Doyon: Département de Mathématiques et de Statistique, Université de Montréal, Montréal, Québec, Canada H3C 3J7

E-mail address: doyon@dms.umontrea1.ca 


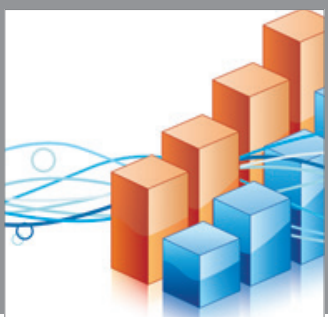

Advances in

Operations Research

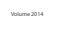

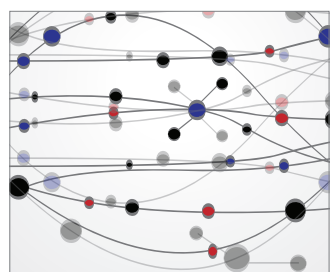

\section{The Scientific} World Journal
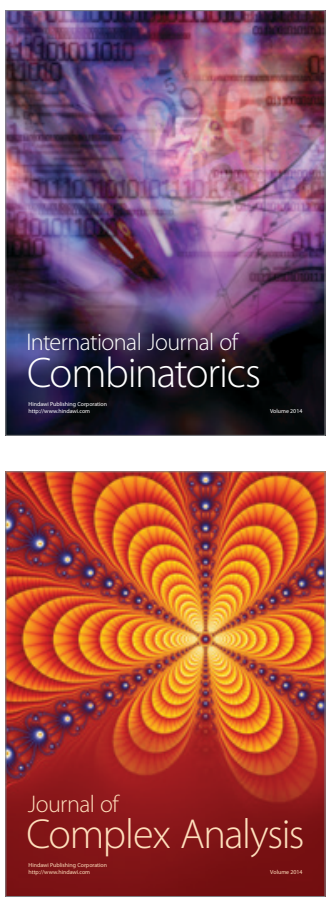

International Journal of

Mathematics and

Mathematical

Sciences
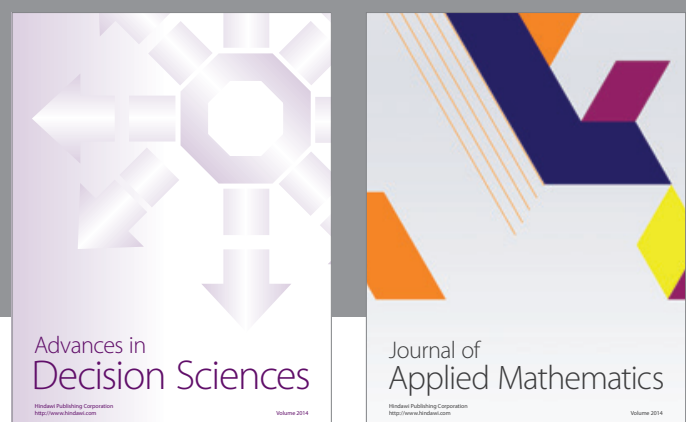

Journal of

Applied Mathematics
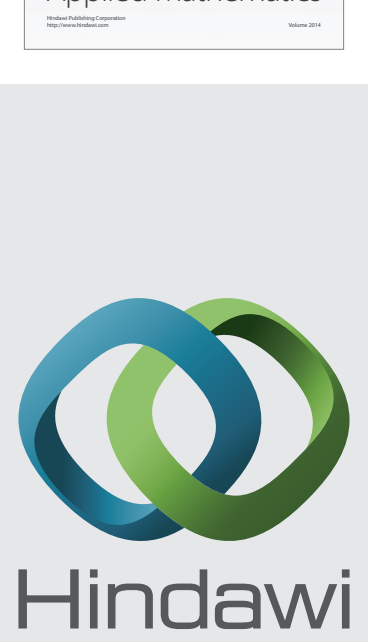

Submit your manuscripts at http://www.hindawi.com
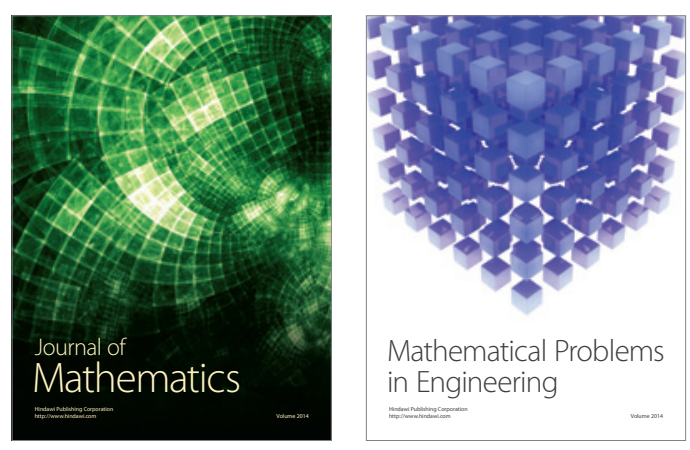

Mathematical Problems in Engineering
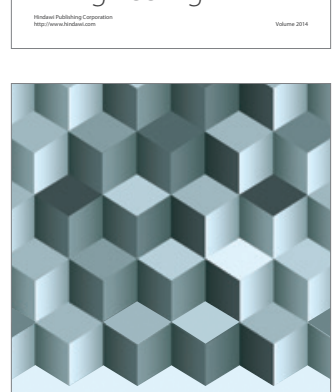

Journal of

Function Spaces
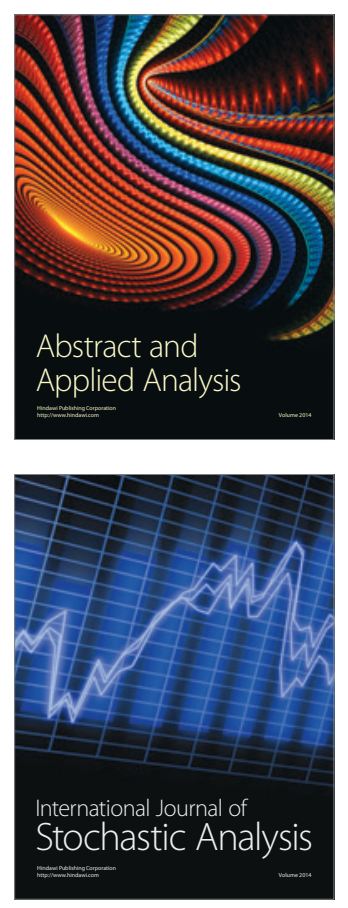

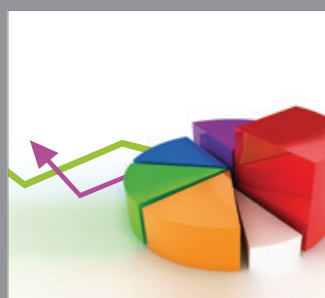

ournal of

Probability and Statistics

Promensencen
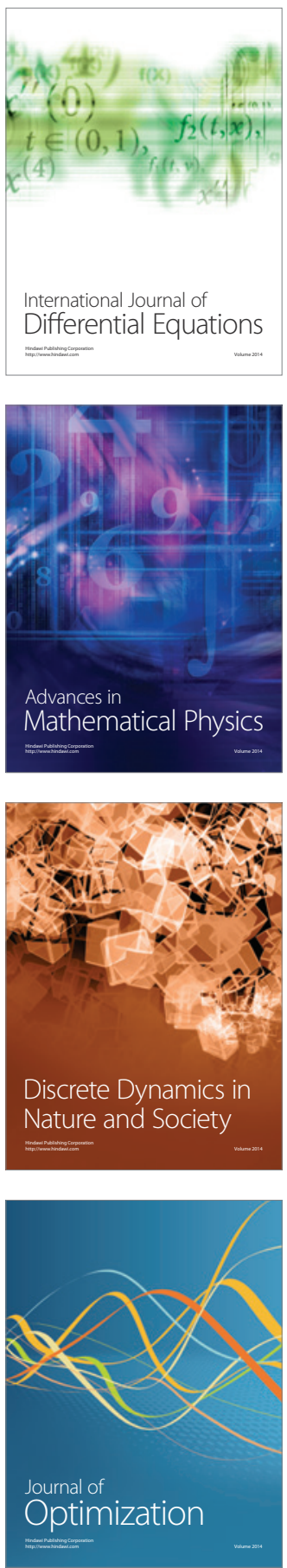